\title{
Fatores Preditores Maternos e Neonatais Relacionados à Prematuridade em um Município do Interior de São Paulo
}

\author{
Maternal and Neonatal Predicting Factors Related to Prematurity \\ in a Countryside City of São Paulo
}

\author{
Isabela de Lemos Duarte ${ }^{1}$ \\ Natália Luiz Neto ${ }^{1}$ \\ Debora Cristina Carretero ${ }^{1}$ \\ Carolina Cristina de Freitas ${ }^{2}$ \\ Adriele Laurinda Silva Vieira ${ }^{3}$ \\ Luana Carolina Rodrigues Guimarães ${ }^{4}$
}

RESUMO

Objetivo: Avaliar os possíveis fatores de riscos maternos e fetais correlacionados aos partos prematuros nascidos no Município de Franca-SP em 2018, incluídos no grupo 10 da classificação de Robson. Metodologia: Trata-se de um estudo epidemiológi$\mathrm{co}$, analítico, observacional e retrospectivo do tipo caso-controle. Considerou-se casos os recém-nascidos pré-termos com idade gestacional inferior a 37 semanas, sendo o feto único com apresentação cefálica, referentes ao Grupo 10 da Classificação de Robson. Como controle, considerou-se os recém-nascidos à termo dos grupos 1, 2, 3, 4, e 5 de Robson. As informações foram coletadas no Sistema de Informação sobre Nascidos Vivos (SINASC), desenvolvido pelo Departamento de Informática do Sistema Único de Saúde (DATASUS). As análises estatísticas foram realizadas considerando-se um nível de significância de 5\%. Resultados: De acordo com o SINASC ocorreram 5.634 partos de nascidos vivos, sendo $10,15 \%$ prematuros, o que corresponde a 572 recém-nascidos do grupo 10 de Robson. O estado civil da mãe, a escolaridade, o número de consultas do pré-natal e a história obstétrica foram fatores preditores. Além disso, o peso ao nascimento, as anomalias congênitas e o apgar do feto também apresentaram influências. A variável fetal de maior predição para parto prematuro foi a existência de anomalias congênitas, visto que estes recém-nascidos tiveram 2,01 vezes mais chances de nascerem prematuros. Conclusão: As condições sociodemográficas, o pré-natal e o estado do feto estão relacionados com a prematuridade.

\section{DESCRITORES}

Feto. Gestação. Parto Obstétrico. Prematuro. Classificação de Robson.

\begin{abstract}
Objective: To evaluate maternal and fetal possible risk factors related to premature births in the city of Franca-SP, included on group 10 of the Robson classification. Metodology: This is an epidemiological, analytical, observational and retrospective case-control study. Preterm newborns with gestational age less than 37 weeks were considered cases, being the only fetus with cephalic presentation, referring to Group 10 of the Robson Classification. As a control, full-term newborns from groups 1 , $2,3,4$, and 5 of Robson were considered. The information was collected from the Information System on Live Births (SINASC), developed by the Informatics Department of the Unified Health System (DATASUS). Statistical analyzes were performed considering a significance level of $5 \%$. Results: According to SINASC there were 5,634 live births, $10.15 \%$ of them were premature, which corresponds to 572 newborns in Robson's group 10. The mother's marital status, education level, number of prenatal consultations and obstetric history were predictive factors. In addition, birth weight, congenital anomalies and fetal apgar score were also influential. The most predictable fetal variable for preterm birth was the existence of congenital anomalies, as these newborns were 2.01 times more likely to be born premature. Conclusion: sociodemographic conditions, prenatal and fetal status are related to prematurity.
\end{abstract}

\section{DESCRIPTORS}

Fetus. Pregnancy. Obstetric Delivery. Premature. Robson's Classification.

${ }_{1}^{1}$ Discente do Curso de Medicina na Universidade de Franca - SP.

${ }^{2}$ Discente do Programa de Pós-graduação em Promoção de Saúde pela Universidade de Franca, Mestre em Ciências pela Universidade de Franca - SP.

${ }^{3}$ Mestre em Ciências da Saúde pela Faculdade de Medicina da Universidade Federal de Uberlândia- MG.

${ }^{4}$ Mestre em Promoção de Saúde pela Universidade de Franca - SP. 
$\mathrm{O}$ parto prematuro requer maior atenção em saúde pública, principalmente em países subdesenvolvidos, em decorrência das condições precárias de saúde da gestante e a alta taxa de morbimortalidade neonatal ${ }^{1}$.

Considera-se como prematuridade o nascimento antes da $37^{a}$ semana de gestação, sendo "prematuros extremos" aqueles nascidos com menos de 28 semanas, "muito prematuro" os que nascem entre 28 e 32 e "moderado prematuro", os nascidos entre 32 e 37 semanas de gestação².

A taxa de prematuridade brasileira, que corresponde a $11,5 \%$, é cerca de duas vezes superior à observada nos países europeus ${ }^{3}$. Nota-se que, em média, $75 \%$ dos casos a gestante inicia espontaneamente o trabalho de parto. Nos $25 \%$ restantes, o parto prematuro é eletivo, isto é, a interrupção da gestação é realizada em decorrência de alguma complicação materna e/ou fetal ${ }^{4}$.

A Classificação de Robson é recomendada pela Organização Mundial de Saúde (OMS), desde 2015, como um instrumento padrão ouro para avaliar, monitorar e comparar as taxas de cesariana em uma mesma ou diferentes instituições, visto que aperfeiçoa o uso das cesáreas ao identificar, analisar e focalizar intervenções em grupos específicos que sejam particularmente relevantes em cada local. Esta classifica todas gestantes, ao internar, em 10 grupos, sendo totalmente inclusiva e mutuamente exclusiva, de modo que todas as gestantes sejam incluídas em apenas um dos 10 grupos, sendo imprescindível para evitar intercorrências perinatais e durante o parto. Essa informação pode ser usada em análises de tendência, verificando se ocorre mudança do perfil ao longo do tempo. De tal forma, grande parte dos pacientes prematuros é abordada no grupo 10 de Robson, visto que este inclui todas as gestantes de feto único com apresentação cefálica e idade gestacional menor que 37 semanas, incluindo aquelas com cesárea anterior, permitindo melhor analise das circunstâncias que levam ao nascimento de recém-nascido pré-termo (RNPT), quando comparado com os demais grupos de Robson. Assim, a avaliação do tipo de amostra ajuda a compreender as características das mulheres da sua população com o uso desta classificação, sendo possível avaliar a qualidade da assistência, das práticas de cuidados clínicos e os desfechos por grupo ${ }^{5,6}$.

Desta forma, o estudo das condições de nascimento infantil no Brasil é necessário para orientar a boa qualidade de atenção nos serviços de saúde, sendo essencial o conhecimento do perfil de nascimento dos fetos prematuros e a frequência dos fatores de risco epidemiológicos, maternos e fetais que contribuem para tal, como idade da mãe, seu estado civil e escolaridade, quantidade de filhos vivos e quantidade de filhos mortos (aborto), duração da gestação, tipo de gravidez e parto, adequação das consultas de pré-natal, apgar, raça/cor, peso ao nascer, presença e tipo de anomalia congênita, quantidade de gestações anteriores, quantidade de partos vaginais anteriores, quantidade de cesáreas anteriores, tipo de apresentação e a idade gestacional ${ }^{7,8,9,10}$.

O objetivo deste estudo foi avaliar os possíveis fatores de riscos maternos e fetais correlacionados aos partos prematuros no Município de Franca, São Paulo, incluídos no grupo 10 da classificação de Robson, identificados pelo SISVAN, e compará-los com os 
recém-nascidos a termo, cefálicos e únicos, do ano de 2018 no Município de Franca.

\section{METODOLOGIA}

Trata-se de um estudo epidemiológico, analítico, observacional e retrospectivo do tipo caso-controle a fim de identificar possíveis fatores preditores para a ocorrência do parto prematuro. A análise foi referente aos nascidos no ano de 2018, no Município de Franca, localizado no interior de São Paulo.

Durante a gestação, a gestante é categorizada mediante a Classificação de Robson, por meio de 6 conceitos obstétricos: paridade da gestante, presença de cesárea anterior, início do trabalho de parto (espontâneo, induzido, cesárea antes do trabalho de parto), idade gestacional, apresentação fetal e número de fetos. Os grupos 1, 2, 3, 4 e 5 incluem os fetos à termo, únicos e cefálicos, variando quanto a paridade, presença de cesárea prévia e início do trabalho de parto. Os grupos 6, 7, 8 e 9 independem da idade gestacional (RNT ou RNPT), variando entre fetos únicos e múltiplos (grupo 8); de apresentação pélvicas (grupos 6 e 7), independente (grupo 8) ou transversa (grupo 9). O grupo 10 identifica os fetos pré-termos, únicos e cefálicos, independente da paridade, cesárea previa e início do trabalho de parto ${ }^{5}$.

Considerou-se como fatores de inclusão os fetos únicos com apresentação cefálica, de modo que os fatores de exclusão correspondem aos recém-nascidos com múltiplos fetos e apresentação pélvica ou transversa. Desta forma, foram considerados 'caso' aqueles recém-nascidos pré-termo de idade gestacional inferior a 37 semanas, referentes ao Grupo 10 da Classificação de Robson. Como 'controle', considerou-se os Recém-Nascidos a Termo (RNT) incluídos nos grupos 1, 2, 3, 4, e 5 de Robson. Serão excluídos da pesquisa os fetos gemelares, incluídos no grupo 8 e aqueles de apresentação não cefálica - grupos 6, 7 e 9.

A relação das informações epidemiológicas foi retirada do Sistema de Informação sobre Nascidos Vivos (SINASC), desenvolvida pelo Departamento de Informática do Sistema Único de Saúde do Brasil (Datasus). Este sistema possibilita traçar o perfil dos nascimentos em cada hospital, município e estado para caracterizar a população.

Como ferramenta, o Datasus criou um programa de domínio público, chamado TabWin , com o intuito de permitir a realização de tabulações rápidas sobre os arquivos que se constituem nos componentes básicos dos sistemas de informações do Sistema Único de Saúde (SUS). Desta forma, para extração dos dados do SINASC, foi necessário realizar o download do programa, disponível no próprio site do Datasus.

Para efetuar as tabulações foram necessários arquivos de definição e de conversão específicos para cada banco de dados. Os arquivos de definição ( ${ }^{*}$.DEF) contêm informações necessárias para identificar quais variáveis estarão disponíveis no painel de controle apresentado pelo programa de modo a possibilitar a tabulação dos dados do respectivo banco. Nos arquivos de conversão ( ${ }^{*}$.CNV) estão as categorias de cada variável do banco de dados e respectivos códigos de identificação. Após download do arquivo foi possível copiá-lo para uma planilha de Excel. 
As variáveis dependentes (variáveis de desfecho) deste estudo foram a ocorrência do parto prematuro (grupo casos) e a ocorrência de partos não prematuros (grupo controles) e o número de semanas da idade gestacional em que o parto ocorreu. As variáveis independentes possíveis preditoras da mãe foram: idade, nível de instrução, raça, estado civil, tipo de gravidez, consultas pré-natais e tipo de parto; quantidade de partos vaginais e cesáreos anteriores, quantidade de filhos vivos e quantidade de abortos prévios. As variáveis independentes preditoras do feto foram o Apgar $1^{\circ}$ minuto, Apgar $5^{\circ}$ minuto, peso ao nascer e presença de anomalias congênitas.

Dentre os classificados no Grupo 10 de Robson, a pesquisa contou com 572 Casos, enquanto o número de Controles correspondeu a 4.642, referentes aos recém-nascidos pertencentes aos grupos 1, 2, 3, 4 ou 5. As perdas justificam-se por serem recém-nascidos de apresentação não cefálica (pélvica ou transversa) e também as pacientes com múltiplos fetos (gemelares), referentes aos grupos 6, 7, 8 e 9 .

Foi sucedida uma comparação entre as frequências das variáveis preditoras qualitativas entre os casos e os controles pelo teste Qui-quadrado. Além disso, foi realizada a análise de regressão logística e a determinação do Odds Ratio (razão de chances) para as variáveis preditoras qualitativas de recém-nascidos pré-termos, com um intervalo de confiança de 95\% (IC95\%). Para o estudo das variáveis preditoras quantitativas realizou-se a comparação entre as médias e medianas dos casos e dos controles pelo Teste T e ANOVA, assim como, uma análise de regressão linear para a idade gestacional de nascimento do neonato (em semanas) dos coeficientes Beta não-padronizados e padronizados. Em todas as análises estatísticas foi considerado o nível de significância de 5\% (com grau de significação de $p<0,05)$, realizadas pelo auxílio do programa computacional SPSS (Statistical Package of Social Science) versão: 20.0.0.

\section{RESULTADOS}

De acordo com os dados disponibilizados pelo SINASC ocorreram 5.634 partos de nascidos vivos no município de Franca-SP, no ano de 2018 , sendo que $10,15 \%$ foram prematuros, ocorrendo antes da $37^{\mathrm{a}}$ semana de gestação, com feto único e apresentação cefálica, correspondendo aos 572 recém-nascidos do grupo 10 de Robson.

A Tabela 1 inclui as variáveis preditoras qualitativas dos casos e dos controles, sendo descrita a comparação da frequência de ambos. Nota-se que apresentaram diferenças estatísticas significativas $(p<0,05)$ pelo teste Qui-quadrado, o estado civil ( $p=0,03)$, em que mães solteiras $(12,48 \%)$ apresentam maior frequência de RNPT em relação às casadas $(9,51 \%)$ ou com união estável $(11,11 \%)$; escolaridade materna $(p=0,001)$, visto que na regularidade de prematuros quanto maior o nível de escolaridade, representando 8,33\% aquelas que estudaram por 12 anos ou mais, contra 19,35\% das mães com 1 a 3 anos de escolaridade; tipo de parto $(p=0,000)$, com maior prevalência de partos vaginais $(13,27 \%)$ quando comparados ao número de cesáreas $(9,77 \%)$; anomalias congênitas ( $p=0,029)$, em que a frequência de $\mathrm{RN}$ prematuros foi maior quando apresentado alguma anomalia congênita $(19,67 \%)$, considerando a frequência de 
Tabela 1. Descrição e comparação entre as frequências das variáveis preditoras qualitativas dos casos (RNPT) e dos controles (RNT)

\begin{tabular}{|c|c|c|c|c|c|c|c|}
\hline \multirow[t]{2}{*}{ Variáveis preditoras } & \multicolumn{2}{|c|}{ Casos } & \multicolumn{2}{|c|}{ Controles } & \multirow{2}{*}{$\begin{array}{c}\text { Total } \\
\mathrm{n}\end{array}$} & \multirow[t]{2}{*}{$\mathrm{X}^{2}$} & \multirow[t]{2}{*}{ Valor-p } \\
\hline & $\mathrm{n}$ & $\%$ & $\mathrm{n}$ & $\%$ & & & \\
\hline \multicolumn{8}{|l|}{ Estado civil materna } \\
\hline Solteira & 296 & 51,75 & 2.075 & 44,76 & 2371 & 12,31 & $0,03^{*}$ \\
\hline Casada & 241 & 42,13 & 2.291 & 49,42 & 2532 & & \\
\hline Viúva & - & - & 06 & 0,3 & 06 & & \\
\hline Divorciada & 21 & 3,67 & 150 & 3,24 & 171 & & \\
\hline União estável & 14 & 2,45 & 112 & 2,42 & 126 & & \\
\hline Ignorada & - & - & 2 & 0,4 & 2 & & \\
\hline Total & 572 & 100 & 4.636 & 100 & 5.208 & & \\
\hline \multicolumn{8}{|l|}{ Escolaridade materna } \\
\hline Nenhuma & - & - & 02 & 0,04 & 02 & 25,30 & $0,001^{*}$ \\
\hline 1 a 3 anos & 06 & 1,05 & 25 & 0,54 & 31 & & \\
\hline 4 a 7 anos & 62 & 10,86 & 363 & 7,82 & 425 & & \\
\hline 8 a 11 anos & 402 & 70,40 & 3.149 & 67,88 & 3.551 & & \\
\hline Maior que 12 anos & 100 & 17,51 & 1.100 & 23,71 & 1200 & & \\
\hline Ignorado & 0001 & 0,18 & - & - & 01 & & \\
\hline Total & 571 & 4.627 & 100 & 5.210 & & & \\
\hline \multicolumn{8}{|l|}{ Raça materna } \\
\hline Branca & 403 & 70,58 & 3.488 & 75,38 & 3.891 & 0,65 & 0,085 \\
\hline Negra & 39 & 6,83 & 254 & 5,49 & 293 & & \\
\hline Amarela & - & - & 02 & 0,04 & 02 & & \\
\hline Parda & 129 & 22,59 & 883 & 19,08 & 1012 & & \\
\hline Total & 571 & 100 & 4.627 & 100 & 5.198 & & \\
\hline \multicolumn{8}{|l|}{ Tipo de parto } \\
\hline Vaginal & 234 & 40,98 & 1.529 & 32,96 & 1.763 & 14,61 & $0,000^{*}$ \\
\hline Cesáreo & 337 & 59,02 & 3.110 & 67,04 & 3.447 & & \\
\hline Total & 571 & 100 & 4.639 & 100 & 5.210 & & \\
\hline \multicolumn{8}{|l|}{ Anomalias congênitas } \\
\hline Sim & 12 & 2,10 & 49 & 1,06 & 61 & 4,79 & $0,029^{*}$ \\
\hline Não & 560 & 97,90 & 4.593 & 98,94 & 5.153 & & \\
\hline Total & 572 & 100 & 4.642 & 100 & 5.214 & & \\
\hline \multicolumn{8}{|l|}{ Consultas pré-natais } \\
\hline Nenhuma & 8 & 1,40 & 27 & 0,58 & 35 & 273,74 & $0,000^{*}$ \\
\hline 1 a 3 & 55 & 9,62 & 137 & 2,95 & 192 & & \\
\hline 4 a 6 & 242 & 42,11 & 875 & 18,85 & 1.117 & & \\
\hline Mais que 7 & 262 & 45,80 & 3.593 & 77,40 & 3.855 & & \\
\hline Ignorado & 5 & 0,87 & 10 & 0,22 & 15 & & \\
\hline Total & 572 & 100 & 4.642 & 100 & 5.214 & & \\
\hline
\end{tabular}

${ }^{*}$ Apresentaram diferenças estatísticas.

Fonte: Sistema de Informação sobre Nascidos Vivos (SINASC) - DATASUS. 
Tabela 2. Análise de regressão logística e determinação do Odds Ratio para as variáveis preditoras qualitativas de recém-nascidos pré-termos

\begin{tabular}{c|c|c|c|c|c|c|c}
\hline Variáveis preditoras & $\boldsymbol{B}_{0}$ & $\begin{array}{c}\text { Erro } \\
\text { padrão }\end{array}$ & $\begin{array}{c}\text { Estatística } \\
\text { Wald }\end{array}$ & $\mathrm{df}$ & $\begin{array}{c}\operatorname{Exp}\left(\beta_{1}\right) \\
\text { OR }\end{array}$ & IC95\% & Valor-p \\
\hline Mãe solteira & $-0,50$ & 0,06 & 11,24 & 1 & 1,32 & $1,11-1,57$ & $0,047^{*}$ \\
\hline $\begin{array}{c}\text { Mãe com baixa esco- } \\
\text { laridade }\end{array}$ & 0,17 & 0,05 & 14,23 & 1 & 0,68 & $0,54-0,86$ & $0,032^{*}$ \\
\hline $\begin{array}{c}\text { Mãe da raça não } \\
\text { branca }\end{array}$ & $-0,23$ & 0,11 & 4,72 & 1 & 0,79 & $0,64-0,98$ & $0,030^{*}$ \\
\hline Parto vaginal & 0,35 & 0,05 & 14,51 & 1 & 1,41 & $1,18-1,69$ & $0,000^{*}$ \\
\hline Anomalias congênitas & 0,70 & 0,03 & 4,60 & 1 & 2,01 & $1,06-3,80$ & $0,032^{*}$ \\
\hline $\begin{array}{c}\text { Efetuar menos que 7 } \\
\text { consultas pré-natais }\end{array}$ & $-0,93$ & 0,55 & 12,19 & 1 & 0,15 & $0,05-0,43$ & $0,000^{*}$ \\
\hline
\end{tabular}

*Apresentaram diferenças estatísticas. Fonte: Sistema de Informação sobre Nascidos Vivos (SINASC) - DATASUS. Legenda: $\aleph_{0}$, coeficiente $ß$ linear; df, graus de liberdade; $\operatorname{Exp}\left(\aleph_{1}\right)$, coeficiente $ß$ de inclinação; OR, Odds Ratio (razão de chances); IC95\%, limites inferiores e superiores do intervalo de confiança a 95\%.

$10,86 \%$ casos que não apresentam malformações; e número de consultas do pré-natal $(p=0,000)$, visto maior prevalência de prematuros quando não realizado um número mínimo de consultas recomendado (superior a $20 \%$ ), com apenas $6,79 \%$ dos casos realizando 7 ou mais consultas.

Quando realizado a análise de regressão logística e de determinação do Odds Ratio das variáveis qualitativas dos casos em comparação com os controles, na Tabela 2, foi possível verificar que apresentaram maiores chance de ocorrência de parto prematuro: mães solteiras $(\mathrm{OR}=1,32$; IC95\% $=1,11$ 1,57 ), mães com baixa escolaridade (OR = $0,68 ; I C 95 \%=0,54-0,86)$, mães que não eram de raça branca $(\mathrm{OR}=0,79 ; \mathrm{IC} 95 \%=$ $0,64-0,98)$, parto vaginal (OR $=1,41$; IC95\% $=1,18-1,69)$, presença de anomalias congênitas $(O R=2,01 ; \mathrm{IC} 95 \%=1,06-3,80) \mathrm{e}$ efetuar menos que 7 consultas pré-natais (OR $=0,15 ; \mathrm{IC} 95 \%=0,05-0,43)$.
Portanto, a determinação do Odds ratio demonstrou que as mães solteiras, com baixa escolaridade, de raça não branca, que tiveram parto vaginal e que efetuaram menos que sete consultas pré-natais tiveram respectivamente 1,$32 ; 0,68 ; 0,79 ; 1,41 \mathrm{e}$ 0,15 vezes mais chances de terem RNPT em comparação aos recém-nascidos a termo. A variável que apresentou a maior predição para parto prematuro foi a existência de anomalias congênitas, visto que estes recém-nascidos tiveram 2,01 vezes mais chances de nascerem prematuros.

Nesta análise, foi possível verificar a influência da raça materna, fato não observado quando realizada a comparação entre a frequência dos RN prematuros e os RN não prematuros, isso porque o teste anterior apenas comparou as frequências e não as predições como na análise de regressão.

As análises das variáveis preditoras quantitativas propostas neste estudo, que 
Tabela 3. Comparação entre as médias e medianas das variáveis preditoras quantitativas dos casos e dos controles

\begin{tabular}{c|ccccccc}
\hline Variáveis preditoras & Grupo & $\begin{array}{c}\text { Média e desvio } \\
\text { padrão }\end{array}$ & Mediana & $V_{\text {Min }}$ & $V_{\text {max }}$ & $\begin{array}{c}\text { Teste de } \\
\text { hipóteses }\end{array}$ & Valor-p \\
\hline Idade materna & Casos & $27,38 \pm 6,27$ & 27 & 13 & 45 & $\mathrm{~T}=1,759$ & 0,078 \\
& Controles & $27,87 \pm 6,27$ & 28 & 14 & 47 & & \\
\hline Peso do recém-nascido & Casos & $2.360,88 \pm 676,60$ & 2.460 & 270 & 4.380 & $\mathrm{~T}=42,470$ & $0,000^{*}$ \\
& Controles & $3.220,43 \pm 421,89$ & 3.210 & 265 & 4.805 & & \\
\hline Quantidade de filhos & Casos & $1,0 \pm 1,18$ & 1 & - & 8 & $\mathrm{Z}=2,289$ & 0,1043 \\
vivos & Controles & $0,89 \pm 1,11$ & 1 & - & 10 & & \\
\hline Quantidade de abortos & Casos & $0,29 \pm 0,59$ & - & - & 4 & $\mathrm{Z}=2,289$ & $0,016^{*}$ \\
e perdas fetais & Controles & $0,22 \pm 0,53$ & - & - & 6 & & \\
\hline Quantidade de partos & Casos & $1,29 \pm 1,45$ & 2 & - & 10 & $\mathrm{Z}=2,368$ & $0,001^{*}$ \\
anteriores & Controles & $1,19 \pm 1,30$ & 1 & - & 10 & & \\
\hline Quantidade de partos & Casos & $0,60 \pm 1,06$ & 1 & - & 10 & $\mathrm{Z}=1,159$ & $0,001^{*}$ \\
vaginais anteriores & Controles & $0,48 \pm 1,02$ & - & - & 7 & & \\
\hline Quantidade de partos & Casos & $0,42 \pm 0,79$ & - & - & 5 & $\mathrm{Z}=1,159$ & 0,246 \\
cesáreos anteriores & Controles & $0,43 \pm 0,68$ & - & - & 6 & & \\
\hline Quantidade de consul- & Casos & $6,04 \pm 8,98$ & 6 & - & 10 & $\mathrm{Z}=16,993$ & $0,001^{*}$ \\
tas pré-natais & Controles & $8,12 \pm 4,78$ & 8 & - & 10 & & \\
\hline Apgar 10 minuto & Casos & $8,04 \pm 5,75$ & 8 & - & 10 & $\mathrm{Z}=12,088$ & $0,000^{*}$ \\
& Controles & $8,71 \pm 2,85$ & 9 & 1 & 10 & & \\
\hline Apgar 50 minuto & Casos & $9,19 \pm 4,02$ & 9 & - & 10 & $\mathrm{Z}=13,010$ & $0,001^{*}$ \\
& Controles & $9,73 \pm 2,35$ & 10 & 2 & 10 & & \\
\hline
\end{tabular}

${ }^{*}$ Apresentaram diferenças estatísticas.

Fonte: Sistema de Informação sobre Nascidos Vivos (SINASC) - DATASUS.

descrevem as características relacionadas à mãe (idade e história ginecológica) e ao recém-nascido (peso ao nascer e apgar no primeiro e quinto minuto), são descritas nas Tabelas 3 e 4 . Na Tabela 3 foi realizada uma comparação entre as médias e medianas dos casos (RN prematuros) e dos controles (RN não prematuros), apresentando diferença estatística significativa as seguintes variáveis: peso ao nascer $(p=0,000)$, com média $2.360,88 \pm 676,6 \mathrm{~g}$ no grupo caso versus a
$3.220,43 \pm 421,89 \mathrm{~g}$ do grupo controle; quantidade de abortos e perdas fetais $(p=0,016)$, em que a média dos casos foi de $0,29 \pm 0,59$ e dos controles foi de $0,22 \pm 0,53$; quantidade de partos anteriores $(p=0,001)$, visto que os RN prematuros apresentaram uma média de $1,29 \pm 1,45$ e os RN não prematuros, uma média de 1,19 $\pm 1,30$ partos anteriores; quantidade de partos vaginais anteriores $(p=0,001)$, em que a média dos casos foi de 0,60 1,06 e dos controles de $0,48 \pm 1,02$; quantidade de 
Tabela 4. Análise de regressão linear das variáveis preditoras quantitativas para a idade gestacional de nascimento do neonato (em semanas)

\begin{tabular}{l|c|c|c|c|c|c}
\hline Variáveis preditoras & $\begin{array}{c}\text { ß não } \\
\text { padronizado }\end{array}$ & $\begin{array}{c}\text { Erro pa- } \\
\text { drão }\end{array}$ & IC95\% & $\begin{array}{c}\text { B } \\
\text { padronizado }\end{array}$ & Teste T & Valor $p$ \\
\hline Idade materna & 0,00 & 0,00 & 0,00 a 0,01 & 0,01 & 0,89 & 0,373 \\
\hline Peso do recém-nascido & 0,39 & 0,04 & 0,29 a 0,48 & 0,62 & 75,15 & $0,000^{*}$ \\
\hline $\begin{array}{l}\text { Quantidade de filhos } \\
\text { vivos }\end{array}$ & $-0,06$ & 0,02 & $-0,11$ a $-0,01$ & $-0,03$ & $-2,50$ & $0,013^{*}$ \\
\hline $\begin{array}{l}\text { Quantidade de abortos e } \\
\text { perdas fetais }\end{array}$ & $-0,28$ & 0,05 & $-0,38$ a $-0,18$ & $-0,08$ & $-5,44$ & $0,000^{*}$ \\
\hline $\begin{array}{l}\text { Quantidade de partos } \\
\text { anteriores }\end{array}$ & $-0,09$ & 0,02 & $-0,13$ a $-0,15$ & $-0,06$ & $-4,38$ & $0,000^{*}$ \\
\hline $\begin{array}{l}\text { Quantidade de partos } \\
\text { vaginais anteriores }\end{array}$ & $-0,02$ & 0,03 & $-0,07$ a 0,03 & $-0,01$ & $-0,79$ & 0,428 \\
\hline $\begin{array}{l}\text { Quantidade de partos } \\
\text { cesáreos anteriores }\end{array}$ & $-0,15$ & 0,04 & $-0,22$ a $-0,07$ & $-0,05$ & $-3,69$ & $0,000^{*}$ \\
\hline $\begin{array}{l}\text { Quantidade de consultas } \\
\text { pré-natais }\end{array}$ & 0,03 & 0,01 & 0,02 a 0,04 & 0,07 & 5,29 & $0,000^{*}$ \\
\hline Apgar 10 minuto & 0,07 & 0,01 & 0,05 a 0,08 & 0,11 & 8,01 & $0,000^{*}$ \\
\hline Apgar $5^{0}$ minuto & 0,08 & 0,01 & 0,06 a 0,10 & 0,11 & 7,74 & $0,000^{*}$ \\
\hline
\end{tabular}

*Apresentaram diferenças estatísticas.

Fonte: Sistema de Informação sobre Nascidos Vivos (SINASC) - DATASUS.

consultas pré-natais $(\mathrm{p}=0,001)$, com média de $6,06 \pm 8,98$ no grupo dos prematuros e $8,12 \pm 4,78$ nos não prematuros; apgar no primeiro minuto $(p=0,000)$, com média dos casos igual a $8,04 \pm 5,75$, contra $8,71 \pm 2,85$ do grupo controle e Apgar no quinto minuto ( $p$ $=0,001$ ), com caso apresentando média de $9,19 \pm 4,02$ e controle, média de 9,73 $\pm 2,35$. Não ocorreram associações significativas entre a ocorrência de parto prematuro e a idade materna, a quantidade de filhos anteriores e a quantidade de partos cesáreas anteriores.

$\mathrm{Na}$ Tabela 4, quando realizada a análise de regressão linear para a idade gestacional de nascimento do neonato (em semanas), todas as variáveis expostas apresentaram diferenças estatísticas significativas, com exceção da idade materna e a quantidade de partos vaginais anteriores ( $p>0,05)$, muito embora sejam fatores reconhecidos como preditores do parto prematuro na literatura científica.

A análise de regressão linear e a determinação do coeficiente beta padronizado demonstrou que a idade materna não foi um preditor para neonatos que nasceram com a idade gestacional reduzida, ou seja, a idade materna não influenciou a idade gestacional $(p>0,05)$. O baixo peso do recém-nascido esteve correlacionado à baixa idade gestacional $(p<0,05)$, mas não pode ser considerada como preditor, visto que é sabido que fetos nascidos com baixa idade gestacional possuem baixo peso (Tabela 04).

A quantidade de filhos vivos, a quantidade de abortos e perdas fetais, a quantidade 
de partos anteriores, incluindo as mães com partos cesáreos tiveram uma correlação negativa e estatisticamente significativa com a idade gestacional $(p<0,05)$, pois o coeficiente beta padronizado demonstrou que o aumento nestas variáveis preditoras pode reduzir o número de semanas gestacionais em $3 \%$, $8 \%, 6 \%$ e $5 \%$, respectivamente. A quantidade de partos vaginais anteriores não esteve correlacionada à baixa idade gestacional dos nascimentos dos neonatos $(p<0,05)$. Portanto, existe indicação de que o parto vaginal seja um fator protetor para a prematuridade (Tabela 04).

Em relação às correlações positivas, foi verificado que quanto maior a quantidade de consultas pré-natais e o Apgar de primeiro e quinto minutos, maior a idade gestacional do recém-nascido, pois estas variáveis influenciaram em $7 \%$ e $11 \%$ (apgar $1^{\circ}$ e $5^{\circ}$ minutos) na idade gestacional do neonato, respectivamente, conforme o coeficiente beta padronizado da análise de regressão linear $(p<0,05)$, como demonstrado na Tabela 04. Assim sendo, também foi possível confirmar que um número aumentado de consultas médicas pré-natais é um fator protetor para a prematuridade.

A idade materna não foi preditora para redução das semanas gestacionais, entretanto, o peso do recém-nascido foi estatisticamente positivo; quanto menor o número de semana, menor é o peso do recém-nascido. No tocante a quantidade de filhos vivos, quanto maior a quantidade de filhos vivos, menor a idade gestacional, isso pode se explicar com as multíparas normalmente tem filhos com idade gestacional menor comparadas às nulíparas. O mesmo pode se descrever em relação aos abortos, quanto maior o número de abortos anteriores, menor será a idade gestacional, $3 \%$ da idade gestacional podem ser explicadas pelos filhos vivos, já a quantidade de aborto foi $8 \%$, visto pelo beta padronizado, a correlação de forma linear dos resultados. $O$ mesmo se repete para quantidade de partos anteriores, para quantidade de partos normais e cesárias anteriores, levando a pensar no caso das multíparas terem historicamente a idade gestacional menor em relação às nulíparas. No entanto, em relação à quantidade de consultas ocorreu o oposto, quanto maior o número de consultas no pré-natal, maior a idade gestacional. O mesmo aconteceu com o apgar; quanto maior a nota do apgar, maior será a idade gestacional, o que também corrobora com os dados da literatura.

\section{DISCUSSÃO}

Estatisticamente, foi possível estabelecer uma estreita relação da prematuridade com diversas variáveis relacionadas à mãe, tais como: estado civil, escolaridade, raça, número de consultas pré-natais, quantidade e tipo de parto assim como a quantidade de abortos/perdas fetais. Além das variáveis relacionadas ao bebê que incluem peso ao nascer e Apgar no $1^{\circ}$ e $5^{\circ}$ minuto $^{11}$.

Dentre as variáveis relacionadas à mãe, acima relatadas, em relação ao estado civil da genitora, o mesmo influenciou no sentido de apontar que as mães solteiras podem ser mais vulneráveis em relação à prematuridade do bebe. Corroborando com essa afirmativa, foi realizado um estudo no Município de Guarapuava/PR e, como resultado, verificou-se que os partos prematuros naquele grupo corresponderam a $51 \%$ de todos aqueles realizados. Dessa maneira, uma possível explicação para essa relação, pauta-se que na ausência de um companheiro é gerado um maior medo, insegurança e 
apreensão na genitora pelo fato da mesma não compartilhar seus anseios, experiências e sentimentos durante a gestação, além do que, existindo um déficit afetivo para essa mãe, ocasionaria um aumento do estresse materno desencadeando, por conseguinte, em um parto antes do período desejado ${ }^{12}$.

A escolaridade materna possui relação de proporcionalidade com o parto prematuro. Assim como consta na literatura, quanto maior o grau de escolaridade menor a taxa de prematuridade. Sabendo que essa variável pode estar diretamente relacionada aos fatores socioeconômicos, a prematuridade, nesse caso, pode ser influenciada pela falta de informações e acesso adequado à saúde para mãe. Firmado a isso, um estudo realizado no Canadá constatou uma queda da prematuridade em mulheres com mais de 17 anos de estudos quando comparado com aquelas que tiverem acesso à educação por tempo inferior a 11 anos ${ }^{13,14}$.

No que concerne à raça das gestantes, de acordo com a amostra realizada, verificou-se um maior risco de prematuridade em grávidas não brancas. Fortalecendo esta afirmativa, em 2003, realizou-se em Londres um estudo no qual se evidenciou uma taxa superior de partos prematuros em mulheres negras e asiáticas presentes naquele grupo. Os resultados podem estar ligados tanto as condições socioeconômicas, como também é capaz de sugerir que o período de maturação fetal pode ser precoce nessas etnias ${ }^{15}$.

Em relação à atenção a assistência pré-natal, as mulheres que não tiveram o devido auxilio apresentaram maior risco de parto prematuro. O mesmo resultado foi encontrado em um estudo realizado no Norte da China, demostrando, assim, a importância da realização do pré-natal para a prevenção da prematuridade. As consultas regulares possuem ampla capacidade de intervir em várias esferas como social, psicológica e instrucional, porém, seu principal foco está na identificação dos fatores de riscos gestacionais, sendo possível encaminhar a parturiente a um serviço especifico. Dessa forma, é possível influir nos vieses que podem ser desencadeantes de um parto precoce ${ }^{12,16}$.

No Brasil, a diretriz do Ministério da Saúde (MS) preconiza o mínimo 6 consultas pré-natais para que seja considerado completo, alegando menores chances de complicações, dentre estas podemos citar a prematuridade, discutida no presente estudo. De acordo com isso, a melhora da quantidade das consultas no pré-natal, pode-se observar melhora em $7 \%$ na prematuridade ${ }^{17}$.

Ainda em relação às variáveis maternas significativas, o tipo de parto vaginal apresenta maior relação com os partos prematuros apesar de existir estudos que ponderam que esta relação esteja mais intimamente relacionada ao parto cesáreo. Quanto aos antecedentes obstétricos, são predisponentes para a prematuridade: a ocorrência de partos vaginais anteriores e a quantidade de abortos e perdas fetais, além da quantidade de abortos e perdas fetais, resultado esse validado também na cidade de Lima, no Peru e em Santa Catarinauma ${ }^{18,19}$.

Ao que concerne aos fatores ligados à criança, o peso ao nascimento mostrou-se em média de $2.360,88 \mathrm{~g}$, de modo que o baixo peso apontou associação direta com menor idade gestacional. Tais resultados também são atestados em outros estudos, como em uma análise do Município de Porto Alegre, em 2012, onde nascidos com peso abaixo 
de $2500 \mathrm{~g}$ apresentaram uma chance quatro vezes maior da ocorrência de parto prematuro; bem como de outro estudo na Cidade de Santa Maria, em que se verificou prematuridade em $78 \%$ das crianças nascidas com baixo peso. Ainda é possível correlacionar o ocorrência de partos prematuros com a presença de anomalias congênitas, dado que também foi observado na Cidade de São Paulo, no período de 2010 a 2014 e no Estado do Rio de Janeiro ${ }^{20,21}$.

Por fim, quando comparado o Apgar entre os grupos caso e controle, constataram-se valores abaixo do índice na análise do grupo 10 de Robson (caso), tanto no primeiro quanto no quinto minuto. O mesmo resultado foi evidenciado em um estudo realizado no Rio de Janeiro e no Município de Maringá/ PR, comprovando, assim, a sua importância e tornando possível prever uma maior morbimortalidade nos RNPT com valores de Apgar baixos ${ }^{22}$.

Outro fator de grande relevância que pode ser levado em consideração como preditor de prematuridade é a idade materna, muito embora, este fator não tenha apresentado correlação expressiva com a ocorrência do parto prematuro na Cidade de Franca-SP, no ano de 2018, divergindo de resultados encontrados em outros estudos, os quais relatam que extremos de idade influenciam na ocorrência de prematuridade ${ }^{23}$.

\section{LIMITAÇÕES DO ESTUDO}

Dentre as limitações do estudo em questão, pode-se citar a dependência de dados disponibilizados pelo DATASUS, visto que a atualização da plataforma influencia sobremaneira na proximidade dos dados em relação ao tempo atual, assim como a oferta de variáveis, em virtude da ausência de condições que interviriam nos resultados da pesquisa.

\section{CONCLUSÃO}

Por meio dos achados neste estudo, concluiu-se que o estado civil da mãe, a escolaridade materna e a história obstétrica influenciaram de maneira significativa a ocorrência de parto prematuro e do baixo peso ao nascimento, podendo ser relacionado à riscos gestacionais, variáveis relacionadas à mãe e ao bebê e associado à condições sociodemográficas. Além disso, quanto mais bem conduzidas as consultas do pré-natal, menor a chance da prematuridade, destacando a relevância de identificação dos fatores de risco para uma adequada intervenção, assim como a melhoria na atenção das políticas públicas de saúde para o enfrentamento do baixo peso ao nascer e parto pré-termo. Pode-se ainda, assegurar de uma melhor condução para os casos de anomalia congênita, outro fator que foi ressaltado como causa de prematuridade do presente estudo. Porém, é importante discutir como tais resultados podem ser ampliados e ou comparados para outras realidades. Deste modo, novos estudos podem ser desenvolvidos a fim de compreender as diferenças e as similaridades entre outros contextos, nas demais regiões geográficas do Brasil ou do mundo. 


\section{REFERÊNCIAS}

1. Sociedade Brasileira de Pediatria. Curvas de crescimento intra-uterino de uma população de alto nível socioeconômico. 2001; 3:1-2

2. World Health Organization. Born Too Soon: The Global Action Report on Preterm Birth. 2012 May;13.

3. Fundação Oswaldo Cruz - FioCruz. Taxa de bebês prematuros no país é quase o dobro do que em países da Europa. [Online].; 2016.

4. Bittar RE CMZM. Condutas para o trabalho de parto prematuro. Rev Bras Ginecol e Obstetr. 2005; 27(9):1.

5. Ministério da Saúde - Fundação Oswaldo Cruz (Fiocruz). CLASSIFICAÇÃO DE ROBSON. [Online].; 2018.

6. Federação Brasileira das Associações de Ginecologia e Obstetricia. Organização Mundial da Saúde (OMS) lança 56 recomendações para tentar diminuir as cesáreas. [Online].; 2018.

7. Ministério da Saúde. A Saúde do Recém-Nascido no Brasil. Atenção à Saúde do Recém-Nascido Guia para os Profissionais de Saúde. 2011, 11-12.

8. American Academy of Pediatrics. The American College of Obstetricians and Gynecologists. Guidelines for Perinatal Care. 2014; 13-15

9. Bittar RE ZM. Qual é a melhor via para de parto para o feto prematuro? Rev Feminina - FEBRASGO. 2010; 38(10): 543-546.

10. NGeC. V. Ministério da Saúde. [Online].; 2018.

11. Almeida ACD. Fatores de risco maternos para prematuridade em uma maternidade pública de imperatriz-ma. Rev Gaúcha de Enfermagem. 2012; 33(2):86-93.

12. Ramos HÂdC. Fatores de risco para prematuridade: pesquisa documental. Rev Anna Nery. 2009; 13(2):297-304.

13. Oliveira LLd. Fatores maternos e neonatais relacionados à prematuridade. Rev Escola de Enfermagem da USP. 2016; 50(3):383-387.

14. Auger N. Contribution of Maternal Age to Preterm Birth Rates in Denmark and Quebec, 1981-2008. Am J Public Health. 2013; 103(10):33-37.
15. Patel RR. Does gestation vary by ethnic group a London-based study of over 122000 pregnancies with spontaneous onset of labour. Int J Epidemiology. 2004; 33(1):107-109.

16. Jiang M. A case control study of risk factors and neonatal outcomes of ;preterm birth. Taiwanese J Obstetrics and Gynecology. 2016; 57(6): 814-818.

17. Ministério da Saúde. Atenção ao pré-natal de baixo risco. Caderno de Atenção Básica. 2013; 32(1):33

18. Ahumada-Barrios ME. Fatores de Risco para parto prematuro em um hospital. Revista Latino-Americana de Enfermagem. 2016; 24(2750):2-6.

19. Grillo E. Fatores de risco para nascimentos prematuros e espontâneos na maternidade do hospital universitário. Repositório Institucional da UFSC. 2015.p-42.

20. Cosme HW. Prevalência de anomalias congênitas e fatores associados em recém-nascidos do município de são paulo no período de 2010 a 2014 . Rev Paulista de Pediatria. 2017; 35(1):34-37.

21. Costa CMS GCLM. Malformações congênitas no Município do Rio de Janeiro, Brasil: prevalência e fatores associados a sua ocorrência. Caderno de Saúde Pública. 2006; 22(11):2423-2424.

22. Cunha AdA. Fatores associados à asfixia perinatal. Rev Bras de Ginecologia e Obstetrícia. 2004; 26(10):799-804.

23. Gravena AAF. Idade materna e fatores associados a resultados perinatais. Acta Paulista de Enfermagem. 2013; 26(10):132-134.

\section{CORRESPONDÊNCIA}

Isabela de Lemos Duarte

Discente do curso de medicina na Universidade de Franca.

Av. Dr. Armando de Sáles Oliveira, 201

Parque Universitário, Franca - SP, 14404-600

E-mail: isaald@outlook.com.br 